\title{
PERFORMANCE EVALUATION OF VARIABLE SAMPLING INTERVALS CONTROL CHARTS BY SIMULATION
}

\section{TARIQ MOHAMMED ALI ALI}

Department of Applied Science, University of Technology, Baghdad, Iraq

\begin{abstract}
The main goal of the wide use of quality control charts is to watch the production process and find any change in the quality of its outputs that can be obtained over time. One of the main defects of Shewhart charts is its relatively slow reaction to the small shifts that occur for the mean of production process ${ }^{[1]}$.Recently, new modifications for the control chart idea have been developed to improve the chart ability; these modifications are based on changing the sampling interval. The basic consideration of variable sampling intervals (VSI) control charts is that after the sample is drawn, the time period for withdrawal of another sample should be short if there is evidence of a change in the production process, and long if there is no evidence of any change. In this research, VSI control chart has been considered for evaluation based on the comparison with the traditional one. Different models have been proposed to study; the pattern of process shift is the main difference between the models of the study, and the simulation used here as an effective tool to evaluate the performance of the VSI control charts. The results of the simulation study are presented and analyzed, If the process shifts are assumed to be a linear time series function, and the time of shift occurrence is zero, then the VSI features will become more effective for the quick detection of process shifts than the traditional fixed sampling intervals (FSI) at low and moderate levels also the conclusions have been drawn and the recommendations for the future work are presented.

KEYWORDS: Quality, Intervals, Shifts, Sampling \& Shewhart
\end{abstract}

Received: Dec 12, 2018; Accepted: Jan 02, 2019; Published: Apr 06, 2019; Paper Id.: IJAERDJUN20191

\section{INTRODUCTION}

Statistical quality control (SQC) can be defined as these statistical methods and engineering ways which are useful in the measurement, monitoring, control, and improvement of quality.

There is a widespread use for control charts and other related techniques to monitor the processes statistically.

Some reasons behind the fame of quality control charts are,

- Control charts are a trustful technique for improvement and development of productivity.

An effective control chart program will reduce and diminish scrap and rework units, which are the main inhibitors of productivity in any process.

- Control charts have a good activity to prevent defects.Quality control charts help to ensure that the production process remains under statistical control, which is consistent with the" do it right the first time", philosophy.

It is never less expensive to separate good products from bad products later, on then it is to build it right 
initially.

If there is not an effective process control, you will pay more money to repair a nonconforming product.

- By control charts we can get information about the capability of process

The control charts provide information about the magnitude of important process parameters and their stability with time; this helps to estimate the process capability.

This Information is of tremendous use to product and process designers.

One of the drawbacks of the Shewhart control chart, however, is that it is a little slow to respond to small shifts or drifts in the process mean. Consequently, a number of alternatives have been proposed to improve upon the performance of the Shewhart chart. Some of these alternatives include Shewhart charts with warning limits, Shewhart charts with runs tests, exponentially weighted moving average chart EWMA, and cumulative sum control charts CUSUM ${ }^{[3]}$.

One of the common features of all control charts mentioned above is that the time between samples from the process is constant ${ }^{[3]}$, this implies that the sampling interval between samples have a fixed length (FSI)

Truth be told, the period of time between two sampling intervals means that there is production output, so if the process mean shifts immediately after the sample taken, then the process will run to the wrong direction for a period of time that may reach to the fixed sampling interval, for this reason the percent of the defective items will increase in the process production.

Recently, there has been extensive advancement in the control chart area, where sampling interval varies relying upon what is observed from the process, any chart uses this thought is called variable sampling interval control chart.

The plan of control charts as for financial criteria has been the subject of impressive investigates amid the most recent three decades.

A lot of various process models have been produced and connected to most of the major types of control charts.

\section{NOMENCLATURE}

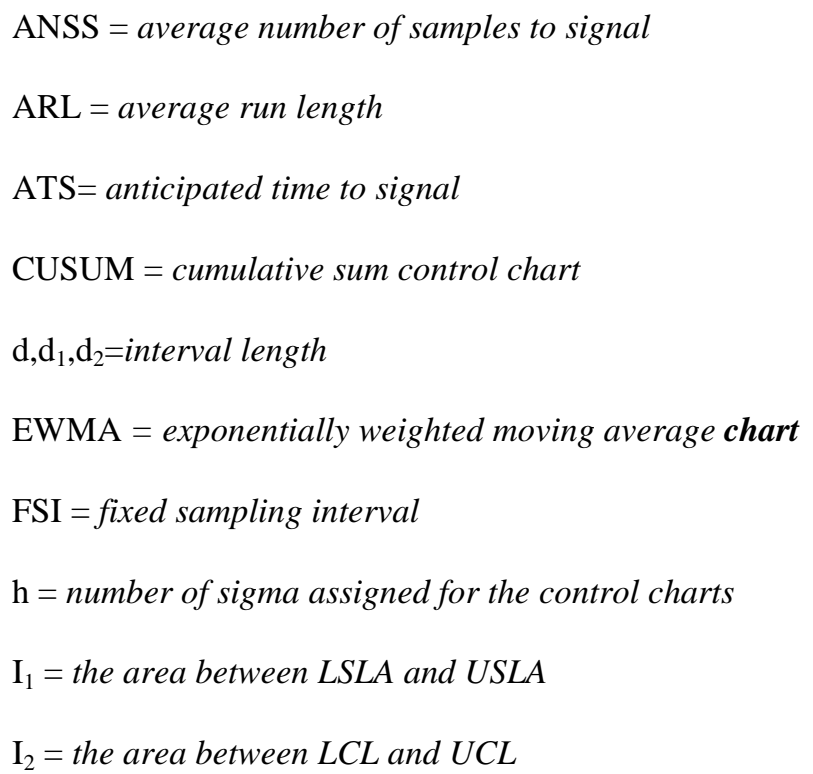


$\mathrm{LCL}=$ lower control limit

LSLA = lower sectional limit area

$\mathrm{n}=$ sample size

$\mathrm{N}=$ number of samples to signal

$\mathrm{p}=$ probability

$\mathrm{q}=$ the probability that the sample falls outside control limits

$\mathrm{q}_{\mathrm{o}}=$ the probability that the sample falls outside control limits at $\mu=\mu_{\mathrm{o}}$

$\mathrm{R}_{\mathrm{i}}=$ sampling interval utilized before the $i^{\text {th }}$ samples are taken

$\mathrm{SQC}=$ statistical quality control

$\mathrm{T}=$ time to signal

$\mathrm{UCL}=$ upper control limit

USLA = upper sectional limit area

VSIFT =variable sampling interval with sampling at fixed times

VSSIFT $=$ variable sample size and sampling intervals at fixed times

\section{Greek Symbols}

$\mu=$ process mean

$\mu_{0}=$ target process mean

$\sigma=$ process standard deviation

$\eta=$ number of intervals

$\theta=$ slope of shift line

$\delta_{\text {in }}=$ shift initial value

$\delta_{\mathrm{t}}=$ process shift amount

\section{THE VARIABLE SAMPLING INTERVALS CONTROL CHART}

The fundamental idea behind variable sampling interval (VSI) control charts is that after a sample is drawn, the time interval until the following sample should be short if there is some sign of process change, and long if there is no indication of process change.

The VSI chart uses a finite number of intervals lengths $d_{1}, d_{2}, d \eta$, where $d_{1}<d_{2}<. .<d \eta$.

The minimum possible length of interval $\mathrm{I}_{\mathrm{t}}>0$ can be determined by physical thought, like the required period for sample taking.

Maximum length of interval $I_{2}$ can be obtained by the greatest value of time that the production authority is 
tending to permit the operation to work without inspection.

Let interval $\left(\mu_{o}-h \sigma / \sqrt{n}, \mu_{o}+h \sigma / \sqrt{n}\right)$ be partitioned into $\eta$ regions $I_{1}, I_{2}, \ldots \ldots, I_{n}^{[4]}$, where $\mu_{o}$ is the target process mean, $\sigma$ is the process standard deviation, and $\mathrm{h}$ is the number of sigma assigned for the control limits.

A case of variable sampling chart(VSI) is appeared in Figure 1, this chart uses two interval lengths, $\mathrm{d}_{1}, \mathrm{~d}_{2}$, as,

$$
\begin{aligned}
& \mathrm{I}_{1}=\left(\mu_{\circ}-\mathrm{h} \frac{\sigma}{\sqrt{\mathrm{n}}}, \mu_{\circ}-\mathrm{h}_{1} \frac{\sigma}{\sqrt{\mathrm{n}}}\right) \cup\left(\mu_{\circ}+\mathrm{h} \frac{\sigma}{\sqrt{\mathrm{n}}}, \mu_{\circ}+\mathrm{h}_{1} \frac{\sigma}{\sqrt{\mathrm{n}}}\right) \\
& \mathrm{I}_{2}=\left(\mu_{\circ}-\mathrm{h}_{1} \frac{\sigma}{\sqrt{\mathrm{n}}}, \mu_{\circ}+\mathrm{h}_{1} \frac{\sigma}{\sqrt{\mathrm{n}}}\right)
\end{aligned}
$$

Where $0<\mathrm{h}_{1}<\mathrm{h}$, represents the number of sigma for the sectional limits area.

Figure 1 explains how VSI control charts attempts to enhance the recognition of X-bar chart, there are three possible decisions can be taken after each sample:

- If $\mathrm{X}$ fell out of control limits, that means a signal

- If $X \in I_{1}$, then take the next sample after $d_{1}$.

- If $\mathrm{X} \in \mathrm{l}_{2}$, then take the next sample after $\mathrm{d}_{2}$.

\section{COMPARISON OF CONTROL CHARTS}

For assessing the benefit of VSI features, it is so normal to do a comparison between performance of the VSI Xbar chart and X-bar chart FSI, at the point when both fixed sampling interval (FSI) and variable sampling interval (VSI) charts have same statistical control limits at $\mu_{\mathrm{o}}+\mathrm{h} \sigma / \sqrt{\mathrm{n}}$. Here, both will have the same magnitude of $\mathrm{q}$ (probability of $X$ falling out control limits).

This indicates the interval employed in VSI chart will not influence the samples number needed to signal, it is advantageous to get the interval utilized by the FSI chart as a time unit, e. g. when the FSI chart gets samples each 2 hours, then the unit of time will have samples every 2 hours period. In such state, the ATS (anticipated time to signal) function numerical magnitude will be similar to the ANSS function numerical magnitude for the FSI chart.

When the VSI chart is put so that the anticipated length of interval is equivalent to a unit of time, if $\mu=\mu_{o}$, then both charts will get the similar ATS functions. At that point, the VSI chart will "correspond" to the FSI chart as in both require a similar samples number to signal, and if $\mu=\mu_{o}$, they get the same rate of average sampling and rate of false-caution through the time.

For this, the ATS functions amounts for both charts can be compared for various $\mu_{1}$ amounts for discovering which chart will make a perfect task to find the variation in $\mu$.

In this study, the VSI control chart will be designed such that it matches with FSI control chart when $\mu=\mu_{\mathrm{o}}$. So, both charts need the similar average samples number of signal, and they have a similar rate of average sampling and false alert through the time at condition of statistical control.

By the constant interval among the samples, the average run length (ARL) can be simply changed to the anticipated time to signal (ATS) by multiplying it with the fixed interval length: 
$\mathrm{ATS}=\mathrm{d} * \mathrm{ARL}$

Where, $\mathrm{d}$ indicates the fixed sampling interval, and ATS is the average samples number to signal.

In this way, ARL can be viewed as the expected time to signal, also, the sampling rate will be constant without taking in the consideration the magnitude of process mean.

Nevertheless, in VSI chart, the sampling rate is determined by the magnitude of $\mu$, and the time to signal is not really a constant multiple of the number of samples to signal ${ }^{[4]}$.

In this manner, for VSI chart, it is important to keep an eye on both samples number to signal and time to signal.

Because the classic idea of the ARL recognizes both number of samples and time, it is therefore preferable to express additional amounts to utilize with VSI control chart.

As far as control limits stay firm, the VSI aspect will get no impact on the likelihood that X-bar drops out of the control limits.

So, the likelihood that $\mathrm{X}$-bar drops out of the control limits is:

$$
\mathrm{q}=\mathrm{p}\left(\overline{\mathrm{x}} \leq \mu_{\circ}-\frac{\mathrm{h} \sigma}{\sqrt{\mathrm{n}}} \text { or } \overline{\mathrm{x}} \geq \mu_{\circ}-\frac{\mathrm{h} \sigma}{\sqrt{\mathrm{n}}}\right)
$$

In any case the interval among the samples, when $\mathrm{N}$ is the samples number to signal, at that point $\mathrm{N}$ possesses a geometric distribution with factor $q$ if the process doesn't change ${ }^{[4]}$.

Thus, ANSS is,

$$
\mathrm{E}(\mathrm{N})=\frac{1}{\mathrm{q}}
$$

And, the variance of $\mathrm{N}$ is:

$\operatorname{VAR}(\mathrm{N})=\frac{1-\mathrm{q}}{\mathrm{q}^{2}}$

At the point, when $\mathrm{T}$ is the time to signal, and $\mathrm{R}_{\mathrm{i}}$ is the sampling interval utilized before the $\mathrm{i}^{\text {th }}$ sample is taken, so:

$\mathrm{T}=\sum_{\mathrm{i}=1}^{\mathrm{N}} \mathrm{R}_{\mathrm{i}}$

Note, practically it may be willing to utilize first the shortest interval $\left(d_{1}\right)$ if the process is just commencing, to provide extra protection against the difficulties that appear during the startup.

The mean time to signal is,

$$
\begin{aligned}
& E(T)=E(N) E\left(R_{i}\right) \\
& E\left(R_{i}\right)=\sum_{j=1}^{\eta} d_{j} \frac{p_{j}}{1-q}
\end{aligned}
$$

$$
\sum_{i=1}^{\eta} p_{i}=1-q<1, \text { thus }
$$

$\operatorname{ATS}=E(T)=\sum_{j=1}^{\eta} \frac{d_{j} p_{j}}{1-q} * \frac{1}{q}$ 
Where, $\eta$ is the number of intervals, the probabilities are condition on no signal, since VSI and FSI control charts are matched at $\mu=\mu_{\mathrm{o}}$, that means:

$$
\mathrm{d} * \mathrm{ARL}=\sum_{\mathrm{j}=1}^{\eta} \frac{\mathrm{d}_{\mathrm{j}} \mathrm{P}_{\mathrm{j}}}{1-\mathrm{q}_{0}} * \frac{1}{\mathrm{q}_{0}}
$$

Where, $\mathrm{q}_{\mathrm{o}}$ is the probability that the sample falls outside the control limits at $\mu=\mu_{\mathrm{o}}$, FSI is the fixed sampling interval and $\mathrm{ARL}=\left(1 / \mathrm{q}_{\mathrm{o}}\right)$, thus,

$$
d=\sum_{j=1}^{\eta} \frac{d_{j} P_{j}}{1-q_{0}}
$$

So, if FSI and VSI control charts are designed such that eq. (11) is satisfied, then the two charts have the same ATS at the state of the statistical control.

For example, if the two have $(3 \sigma / \sqrt{\mathrm{n}})$ control limits, then $1 / \mathrm{q}_{\mathrm{o}}=370.37$ sample, and at 1-hour fixed sampling interval, the ATS for the FSI is 370.37 .

So, for VSI to be matched with FSI of 370.17 average run length at $\mu=\mu_{\circ}$, eq. (11) should be satisfied, so:

$$
\sum_{j=1}^{\eta} d_{j} p_{j}=0.9973
$$

If the VSI control chart has two sampling intervals $(0.1,1.9)$ hour with equal probabilities, eq. (11) is achieved. So by substituting in (10),

$$
\mathrm{ATS}=\frac{0.1 * 0.49865+1.9 * 0.49865}{1-0.0027} * \frac{1}{0.0027}=370.37
$$

So $(0.1,1.9)$ VSI control chart with equal probabilities $\left(1-\mathrm{q}_{\mathrm{o}} / 2\right)$ is matched to FSI control chart of 1-hour fixed sampling interval at condition of statistical control.

\section{VSI PERFORMANCE MEASUREMENT}

The control chart characteristics are dictated by the time length that takes the chart to make a signal.

When the process is statistically controlled, then this time must be long, so the false alarms rate is low. But, when the processes mean shifts, in this case, the time between shift and signal must be short, so the detection occurs quickly.

Samples number before the signal is usually termed in literature of quality control as run length, and the anticipated samples number to signal is termed as average run length (ARL).

Besides FSI, the average run length ARL can be simply changed to the anticipated time to signal by multiplying it with the fixed interval length, so that the average run length can be considered as the anticipated time to signal.

Additionally, the rate of sampling will not vary without concerning the process mean $\mu$ magnitude.

But with VSI chart, nevertheless, the time to signal is not a constant multiple of samples number to signal, and the rate of sampling relies on the $\mu$ value ${ }^{[4]}$.

So, in VSI charts, it is important to develop isolate measures for number of samples and for time. 
The words created in the VSI papers are ANSS (average number of samples to signal) and ATS (average time to signal), also ATS refer to primary measurement of chart ability to detect the shifts in $\mu$

This computation is frequently based on the assumption that the shift in $\mu$ occurs before the first sample is taken.

\section{SIMULATION WORK AND ANALYSIS}

This research provides an evaluation of the performance of the VSI as compared to the traditional FSI.

This comparison study is mainly based on the Simulation approach, as the tool for estimating the parameters required for the comparison.

At this stage, we have to answer the following questions: What is to be evaluated against? How about process shift distribution? When do shifts occur?

The first question is related to determine whether the situation is based on the evaluation of the standard variable sampling interval idea or one of its modifications (VSIFT or VSSI).

Standard Shewhart chart, CUSUM chart or EWMA chart can be considered as the answer of the second question.

In the literature, samples have been treated as independent, i.e., the effect of autocorrelation between the samples is negligible. In each situation, we have to decide whether this assumption is to be considered.

According to the literature, the shift in process mean is assumed to be unchanged.

This implies that the incident of an assignable cause makes a shift in the mean of process which stays constant till detecting and removing the assignable cause.

Gradual mean drift far from the objective value is another kind of shifting; such drift can be continued till it is discovered and removed. In particular processes, this shift can be significant to a certain extent, like the tool wear in metal cutting processes.

Dealing with process shift as a time series function has been considered to evaluate the VSI idea in the case study.

In some situations, process is assumed to be shifted as soon as the monitoring process has started. In some applications, process starts with the state of statistical control and then shifts in a random point of time in the future.

In this research, the situation where the shift occurs as the process starts will be considered in all the case studies.

Simulation procedures will be considered for different situations for the purpose of:

- Comparing the performance of matched FSI and VSI control charts.

- Studying the effect of the spacing between the sampling intervals on the chart efficiency.

In this case, four different situations are proposed to simulate, the main difference between these situations is the symmetry state of the subintervals around the fixed interval and the time of taking the first sample. These different situations can be abstracted in Table 1.

Evaluation the Performance of VSI Control Charts When the Process Shifts as Time Series Function and Occurs at Time Equal to Zero

Let us consider the situation where the control chart is used to monitor the production process for the purpose of 
determining if the process is statistically controlled, changing the process from an out-of-control case to a control state as well as monitoring the process to ensure that it stays in control.

The efficiency of the chart is measured in terms of duration of time that the control chart will get for detecting the process shift, a system such that can be called a quality control system.

Monitor the quality level of the process is done by taking a sample data from the process and computing the sample statistic, which is a random variable. For this reason, the quality control system considered here can be described as a probabilistic system.

For more explanation of this system, a number of terms have to be defined in entity is an object of interest in the system, such as a product, sampling intervals, etc.

In attribute is a property of an entity, for example quality statistic, and variability of sampling intervals. An activity represents a time period of specified length, collecting sample data can be considered an activity and calculating the sample statistic.

The state of a system is defined by the collection of variables necessary to describe the system at any time, the time since the monitoring starting, number of samples, and the amount of process shift.

\section{DESCRIBING THE SIMULATION MODEL}

$\mathrm{X}$-bar control chart is used to monitor the process quality level, and in this chart, process observations distribution is natural in relation with the mean $\mu$ and certain variance $\sigma^{2}$.

Where,

$\mu_{\mathrm{o}}$ : mean objective value.

Assuming that the random samples of size (n) are drawn from the process at each sampling point, and let:

$\mathrm{Xi}=\left(\mathrm{Xi}_{1}, \mathrm{Xi}_{2}, \ldots \ldots, \mathrm{Xi}_{\mathrm{n}}\right)$ represents the sample drawn at the $\mathrm{I}^{\mathrm{th}}$ point.

If $\mathrm{i}^{\text {th }}$ sample is drawn, the sample mean (Xi) is calculated and drawn on a control chart having a center line $\mu_{\circ}$ and control limitsat $\left(\mu_{\mathrm{o}} \pm \mathrm{h \sigma} / \sqrt{ }(\mathrm{n})\right.$, where $\mathrm{h}$ is the number of sigma taken for the control limits.

Then for each sample, the decision rules used after $\mathrm{i}^{\text {th }}$ sample are,

- If X-bar is outside the control limits, then signal;

- If USLA $\leq \mathrm{X}<\mathrm{UCL}$ or LSLA $\geq \mathrm{X}>\mathrm{LCL}$, then take the next sample after $\mathrm{d}_{1}$;

If no one of the above, then take the next sample after $\mathrm{d}_{2}$.

- If the false alarm occurs, then the next sample will be taken after $d_{1}$.

Where,

USLA: upper sectional limit area

LSLA:lower sectional limit area

In fact, this model is based on the assumptions that observations are independent and the shift in the mean of 
process occurred at the beginning.

Since the shift occurs, it is continually changing over time according to a linearly time series function such that at any point of time, the amount of shift in the process can be computed as follow,

$$
\delta_{\mathrm{t}}=\delta_{\text {in }}+\theta\left(\mathrm{t}_{\mathrm{s}}\right)
$$

Where, $t$ represents the time of taking the current sample, $\theta$ is the slope of shift line, $\delta_{\text {in }}$ represents the initial value of shift or the shift at the time equal to zero.

The speed, with which a control chart detects the process mean shifts, measures its statistical efficiency.

The model described above has been converted to run on computer by using a MS-Basic Computer Program (Beginners All-purpose Symbolic Instruction Code).

\section{COMPUTER PROGRAM DESCRIPTION}

\section{Data Input}

The program is designed such that the user specifies all the necessary data for the simulation process.

These data input are, such as the number of sigma for the control limits (h), sample size (n), the fixed sampling intervals (FSI), the initial shift value (delta0), and the variable sampling intervals $\left(\mathrm{d}_{1}, \mathrm{~d}_{2}\right)$.

For more flexibility, the program is designed such that the user has the ability to specify the length before the first sample taking.

As the user enters all the data, the program calculates the other chart parameters, such as the control limits and the sectional limits areas, and then prints out on the screen all the data that were entered for the purpose of checking before starting the simulation study.

\section{Data Treatment}

This computer program treats the sample average as a normal random variable and generates its values using the direct transformation technique.

At each sampling time, the program calculates the amount of shift in the process by using equation (2.1), then calculates the process mean as $\mu_{1}=\mu_{\mathrm{o}}+\mathrm{h} \sigma$.

The direct transformation technique is used again to generate the sample average at this point for normal distribution which has the parameters $\left(\mu, \sigma_{\mathrm{x}}\right)$.

For simplicity, the traditional fixed sampling interval control chart is treated as a special case of the VSI when the two sampling intervals equal the fixed interval.

The computer program is designed to give a different random number seed at each run time, where the randomize statement is connected with a timer.

If one needs to run the computer program at certain seed, he has to delete the timer and write the required seed number in the list of the computer program. 


\section{Information Output}

The computer program prints out the information outputs, and during the run, the program prints out the information that describe the state of the system at each run in each group.

When the simulation study ends, the program prints out the information that represent the estimated values of the necessary parameter (average time to signal) for the different groups and the over-all ATS.

\section{COMPUTER CODE VERIFICATION}

In fact, this simulation model can be converted to other different models according to the values of shift equation parameters, this property will be used for the purpose of verifying the computer code.

Let us suppose the initial shift has a specified value, then this means that the shift in the process occurs at a future point of time and as it occurs it stays at its initial value until detected and eliminated.

In fact, this model is considered before, and the numerical results are presented in the previous researches.

The shift equations of this situation are presented as follow,

$\delta_{\mathrm{t}}=\delta_{\text {in }}$

As a step of code verification, the computer program again runs at this situation.

The simulation results are presented below in table 2 .

Now after the model has been described and the code has been verified, the simulation study for the current case study is started at the different situations.

The results of this study are presented in the following tables.

Three different VSI control charts symmetric around the fixed interval of one hour have been simulated, and the results are presented in Table 3.

The simulation has been done at three different levels of the slope of the shift, small, moderate and large.

From Table 3, one can clearly note that the variable sampling interval control chart performs better than the traditional one in process shift detection, especially in the case of the low and moderate shift.

In this case, the assumption of normally distributed sample average is proposed.

When the assignable cause occurs, the amount of shift is assumed to be changed according to a linear time series function, and this shift stays constant at this value until the assignable cause is detected and eliminated.

Now, let us consider the situation of symmetric VSI control charts, where the first sample is taken after the minimum sampling interval, table 3 shows the ATS as a result of simulating these charts against the FSI control chart at three different levels of shift line slope.

In table (3), three different levels of shift have been considered, small, moderate and large.

At this situation, one can note that the VSI control chart still keeps its advantage in the quick detection of process shift, especially in the low and moderate levels of the process shift. 
As the slope of the shift line increases, the performance of the traditional control chart becomes close to the VSI.

The results of the simulation of this situation are plotted, as shown in Figure 2.

Based on the current study objectives, the next situation is proposed. In this situation, we are going to study the effect of spacing between the sampling intervals of the VSI control chart, according to that three different spacing variable sampling intervals control charts simulated against the FSI control chart, where each chart takes the first sample after its minimum sampling interval. Results of this study are shown in Table 4 and plotted in Figure 3.

From table 4, can note that the VSI control chart still maintains its advantage in the quick detection of process shift at low and moderate shift levels, this is what can be concluded from the results shown in the above table.

But at the large shift levels, we note that the VSI control chart loses its advantages, such that the FSI may take value of ATS less than that of VSI, this difference increases as the two sampling intervals spacing far apart.

Figure 2 shows the result of simulating three asymmetric control charts having the same minimum sampling interval. In this situation, all the charts have the same shift value at the end of the first sampling interval, such that increasing the space between the sampling intervals will effect on the ATS value at the large shift values, as the two sampling interval increased, the chart performance will be decreased.

In third and fourth situations of this case, all the charts are assumed wait equal time until we take the first sample; this interval is selected to be the fixed interval of the fixed interval control chart.

Table 5 represents the ATS as a result of simulating three symmetric VSI control charts. The results show that the VSI control chart is still faster than the traditional one in process shift detection at low and moderate shift levels but at large shift values, the VSI loses its feature in the quick detection as the two sampling intervals spaced far apart.

Table 5 and Figure 4 show the result of this simulation study.

Table 6 represents the result of simulating three VSI control charts having the same minimum sampling intervals. These charts are simulated again based on the assumption that the first sample is taken after the fixed sampling interval. VSI has the same performance of the previous situation; Figure 5 shows the result of this simulation study.

\section{CONCLUSIONS AND RECOMMENDATIONS}

- If the process shifts are assumed to be a linear time series function, and the time of shift occurrence is zero, then the VSI features will become more effective for the quick detection of process shifts than the traditional FSI at low and moderate levels.

- The idea of varying the sampling intervals within a fixed interval has proved its ability to reduce the time of detecting the process shift VSIFT control chart (variable sampling interval with sampling at fixed times).For future work, a new modification for the VSSIFT (variable sample size and sampling intervals at fixed times) idea can be considered.

- In this adjustment, samples are constantly drawn at same settled intervals, similarly dispersed time points, but additional samples are permitted between these time points whenever there are some evidences of a process change. 
- Then for each sample, the decision rules to use after the current sample are,

- If $\mathrm{X}$ fell outside the control limits, then signal.

- If $\mathrm{X} \in \mathrm{I}_{1}$, then take the next sample after $\mathrm{d}_{1}$.

If $\mathrm{X} \in 1_{2}$, then take the next sample after $\mathrm{d}_{2}$

- Any chart using this modification can be called variable sample size sampling interval with fixed time VSSIFT. The justification of this is that when the parameter $\mathrm{g}$ (the limit separating between the two areas $\mathrm{d}_{1}, \mathrm{~d}_{2}$ ) is selected to be close to the control limits, the VSIFT works most of the time as the traditional FSI chart.

- The subject of the trade-off between large sample size (high cost) and the minimizing of the detection time (saving) needs more investigations in future works in this area.

- Most of the researches in a field of VSI control charts and its modifications are mainly done in the area of variable control charts, attribute control charts may be considered for evaluation in future works.

\section{REFERENCES}

1. Montgomery, D. C., “A Discussion on Statistically-Based Process Monitoring and Control”, Journal of Quality Technology, 1997, Vol.29. pp.121.

2. Montgomery.D. C., "Introduction to Statistical Quality Control”, 2nd Edition, John Wiley \& Sons, 1991.

3. Ranger G. C.; and Pignatiello J. J., "Adaptive Sampling for Process Control“, Journal of Quality Technology, 1991, Vol.23, pp.135-155.

4. V. Jemmy Joyce et al., Designing and Selection of Reliability Based Sampling Plans, International Journal of Mechanical and Production Engineering Research and Development (IJMPERD) 2018, Vol. 8(4), pp. 343-348

5. Reynolds M. R., Amin R. W.; Arnold J. C., and Nicholas J. A., "X-bar Charts with Variable Sampling Intervals, Technimetrics“, 1988, Vol.30, pp.181-192.

6. Reynolds, M. R., "Shewhart and EWMA Variable Sampling Interval Control Charts with Sampling at Fixed Times", Journal of Quality Technology, 1996, Vol.28, pp.199-212

7. Albin S. L., Kang S. G., “An X-bar and EWMA Chart for Individual Observation”, Journal of Quality Technology, 1997, Vol.29, pp.41-48.

8. Box G.E, Coleman, D. E, Baxely R.V., “ A Comparison of Statistical Process Control and Engineering Process Control", Journal of Quality Technology,1997,Vol29,pp. 128-130 
Tables and Figures

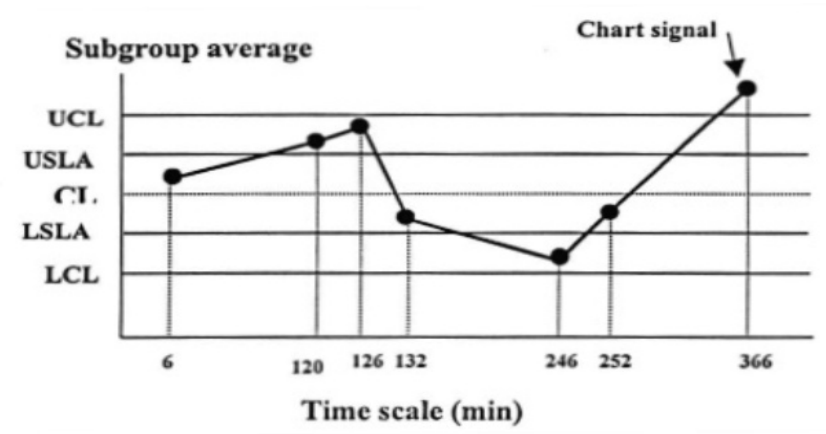

Figure 1: Variable Sampling Interval X-bar Chat $(d 1=6, d 2=114)$ [4]

Table 1: The Different Situations Proposed to Study

\begin{tabular}{|c|c|c|c|c|}
\hline Case \# & Shift pattern & Situation & The state & Taking the first sample \\
\hline \multirow{3}{*}{1} & \multirow{3}{*}{1} & 1 & Symmetric & After $d_{1}$ \\
\cline { 3 - 5 } & Time series function & 2 & Asymmetric & After $d_{1}$ \\
\cline { 3 - 5 } & (Linear) & 3 & Symmetric & After the fixed interval \\
\cline { 3 - 5 } & & 4 & Asymmetric & After the fixed interval \\
\cline { 3 - 5 } & & &
\end{tabular}

Table 2: Simulation Results Model

\begin{tabular}{|c|c|c|}
\hline \multirow{2}{*}{$\delta_{\text {in }}$} & Previous results & Simulated Results \\
\cline { 2 - 3 } & $\left(\mathrm{d}_{1}, \mathrm{~d}_{2}\right)=(0.1,2.8), \mathrm{hr}$ & $\left(\mathrm{d}_{1}, \mathrm{~d}_{2}\right)=(0.1,2.8), \mathrm{hr}$ \\
\hline 0.5 & 21.22 & 21.30 \\
\hline 1.00 & 2.04 & 2.13 \\
\hline 1.25 & 1.50 & 1.57 \\
\hline 1.5 & 1.37 & 1.39 \\
\hline
\end{tabular}

Table 3: ATS of Symmetric VSI Control Chart Matched to FSI Control Charts, First Sample Taken after $d_{1}$, Process Shifts Linearly

\begin{tabular}{|c|c|c|c|c|}
\hline \multirow{2}{*}{ Slope } & \multirow{2}{*}{$\begin{array}{c}\text { FSI } \\
\text { 1-hour }\end{array}$} & \multicolumn{3}{|c|}{ VSI } \\
\cline { 3 - 5 } & $\mathbf{( 0 . 5 , 1 . 5 )}$ & $(0.3,1.7)$ & $(0.1,1.9)$ \\
\hline $\mathbf{0 . 0 0 5}$ & 133.89 & 129.92 & 128.26 & 126.566 \\
\hline $\mathbf{0 . 0 1 0}$ & 89.45 & 86.143 & 84.657 & 83.302 \\
\hline $\mathbf{0 . 1 0 0}$ & 18.45 & 17.043 & 16.397 & 15.835 \\
\hline $\mathbf{0 . 5 0 0}$ & 5.522 & 4.994 & 4.778 & 4.614 \\
\hline $\mathbf{1 . 0 0 0}$ & 3.282 & 2.969 & 2.831 & 2.673 \\
\hline 1.500 & 2.436 & 2.213 & 2.206 & 2.169 \\
\hline
\end{tabular}




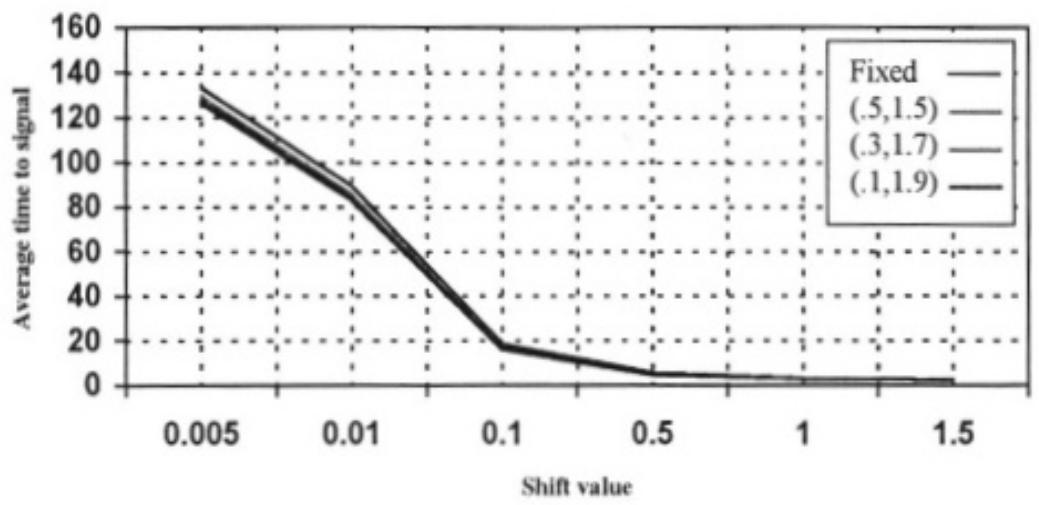

Figure 2: ATS against Process Shift for Symmetric VSI Control Chart Matched to FSI, First Sample Taken after $d_{1}$, Process Shifts Linearly

Table 4: ATS of Asymmetric VSI Control Charts Matched to FSI Control Charts, First Sample Taken after $d_{1}$, Process Shift Linearly

\begin{tabular}{|c|c|c|c|c|}
\hline Slope & \multirow{2}{*}{$\begin{array}{c}\text { FSI } \\
\text { 1-hour }\end{array}$} & \multicolumn{3}{|c|}{ VSI } \\
\cline { 3 - 5 } & $(0.3,1.5)$ & $(0.3,3.0)$ & $(0.3,5.0)$ \\
\hline $\mathbf{0 . 0 0 5}$ & 133.88 & 128.58 & 128.09 & 128.01 \\
\hline $\mathbf{0 . 0 1 0}$ & 89.446 & 84.99 & 84.54 & 84.50 \\
\hline $\mathbf{0 . 1 0 0}$ & 18.44 & 16.49 & 16.46 & 16.40 \\
\hline $\mathbf{0 . 5 0 0}$ & 5.532 & 4.790 & 4.930 & 5.62 \\
\hline 1.000 & 3.284 & 2.834 & 2.870 & 2.93 \\
\hline 1.500 & 2.435 & 2.140 & 2.610 & 2.89 \\
\hline
\end{tabular}

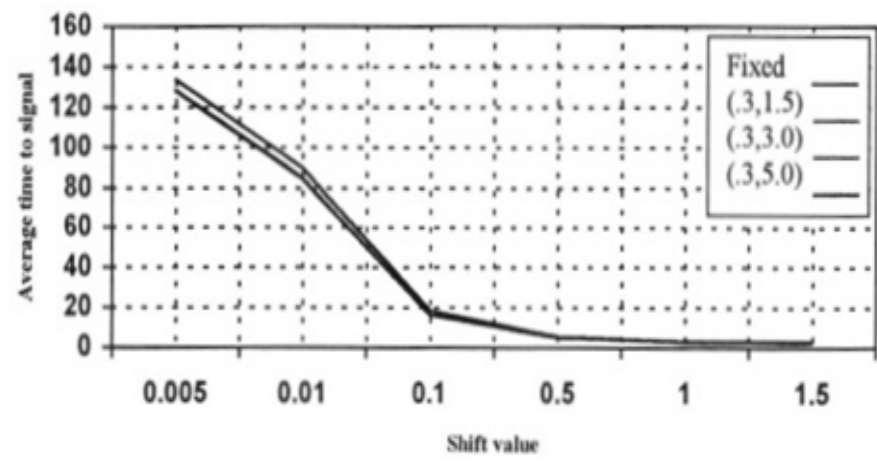

Figure 3: Simulated ATS against the Process Shift for an Asymmetric VSI Control Chart Matched to FSI, First Sample Taken after $d_{1}$, Process Shifts Linearly 
Table 5: ATS of Symmetric VSI Control Chart Matched to FSI Control Charts, First Sample Taken after Fixed Interval, Process Shifts Linearly

\begin{tabular}{|c|c|c|c|c|}
\hline Slope & FSI & \multicolumn{3}{|c|}{ VSI } \\
\cline { 3 - 5 } & 1-hour & $(0.5,1.5)$ & $(0.3,1.7)$ & $(0.1,1.9)$ \\
\hline 0.005 & 133.90 & 130.313 & 128.424 & 126.764 \\
\hline 0.010 & 89.91 & 86.234 & 84.772 & 83.501 \\
\hline 0.100 & 18.46 & 17.056 & 16.471 & 15.851 \\
\hline 0.500 & 5.522 & 4.998 & 4.805 & 4.628 \\
\hline 1.000 & 3.278 & 2.990 & 2.882 & 2.917 \\
\hline 1.500 & 2.442 & 2.171 & 2.098 & 2.165 \\
\hline
\end{tabular}

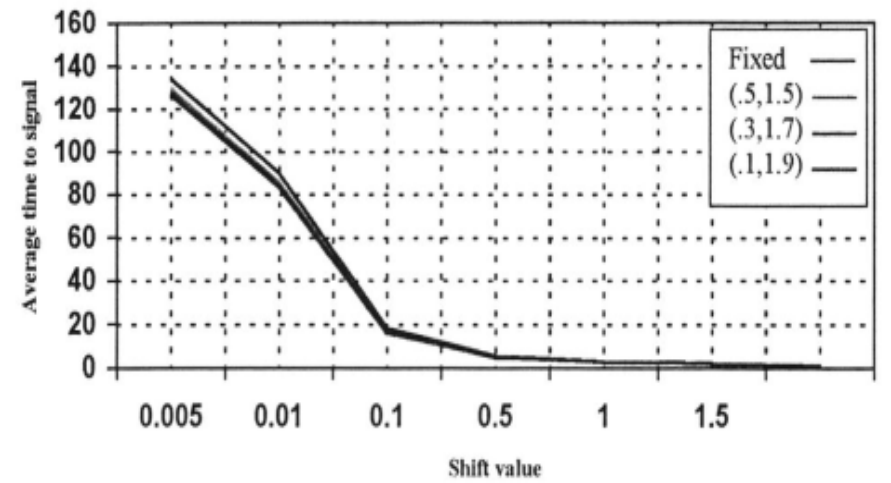

Figure 4: Simulated ATS against the Process Shift for a Symmetric VSI Control Chart Matched to FSI, First Sample Taken after Fixed Interval, Process Shifts Linearly

Table6: ATS of Asymmetric VSI Control Charts Matched to FSI Control Chart, First Sample Taken after Fixed Interval: Process Shifts Linearly

\begin{tabular}{|c|c|c|c|c|}
\hline \multirow{2}{*}{ Slope } & FSI & \multicolumn{3}{|c|}{ VSI } \\
\cline { 3 - 5 } & 1-hour & $\mathbf{( 0 . 3 , 1 . 5 )}$ & $\mathbf{( 0 . 3 , 3 . 0 )}$ & $\mathbf{( 0 . 3 , 5 . 0 )}$ \\
\hline $\mathbf{0 . 0 0 5}$ & 133.91 & 129.54 & 128.14 & 128.09 \\
\hline $\mathbf{0 . 0 1 0}$ & 89.911 & 85.09 & 84.99 & 84.61 \\
\hline $\mathbf{0 . 1 0 0}$ & 18.474 & 16.52 & 16.50 & 16.45 \\
\hline $\mathbf{0 . 5 0 0}$ & 5.531 & 4.91 & 4.99 & 5.76 \\
\hline $\mathbf{1 . 0 0 0}$ & 3.283 & 2.90 & 2.99 & 3.06 \\
\hline $\mathbf{1 . 5 0 0}$ & 2.444 & 2.18 & 2.72 & 3.01 \\
\hline
\end{tabular}

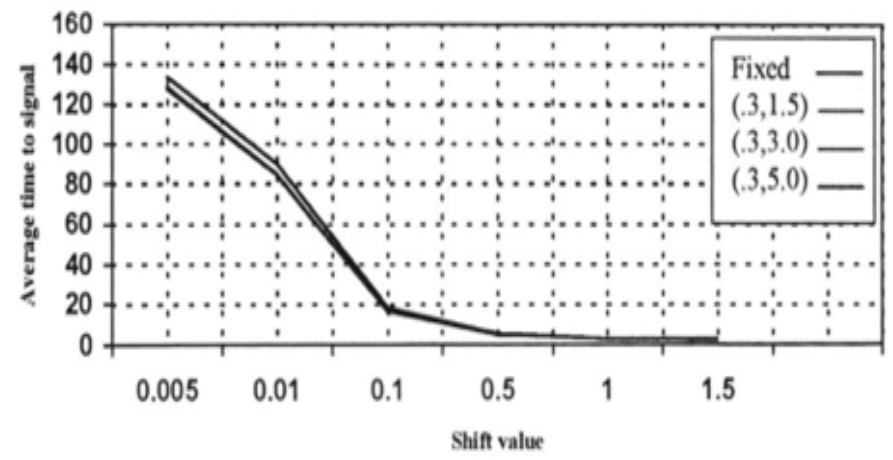

Figure 5: Simulated ATS against the Process Shift for Asymmetric VSI Control Chart Matched to FSI, First Sample Taken after Fixed Interval, Process Shifts Linearly 
